\title{
Características bucais e manejo com comportamental de pacientes com Síndrome de
}

\section{Down}

\section{Dental characteristics and behaviorall management for Down Syndrome patients}

Recebido: 08/11/2020 | Revisado: 15/11/2020 | Aceito: 02/12/2020 | Publicado: 08/12/2020

\author{
Asuka Usui \\ Centro Universitário das Faculdades Metropolitanas Unidas, Brasil \\ E-mail: asuka-usui@hotmail.com \\ Dayane de Moura Campos \\ Centro Universitário das Faculdades Metropolitanas Unidas, Brasil \\ E-mail: dayane.moura63@gmail.com \\ Caleb Shitsuka \\ Centro Universitário das Faculdades Metropolitanas Unidas, Brasil \\ E-mail: cashitsuka@gmail.com \\ Irineu Gregnanin Pedron \\ Universidade Brasil, Brasil \\ E-mail: igpedron@alumni.usp.br \\ Ricardo Shitsuka \\ Universidade Federal de Itajubá, Brasil \\ E-mail: rshitsuka@yahoo.com
}

\begin{abstract}
Resumo
A Síndrome de Down é uma das alterações cromossômicas e a anomalia genética mais frequente.Os indivíduos portadores desta síndrome possuem inúmeras alterações bucais e funcionais que poderão ter consequências nos cuidados de saúde e higiene oral. Existem várias doenças sistêmicas associadas à essa síndrome, tornando-se importante perceber quais as repercussões destas na cavidade oral e no tratamento odontológico. Pela precária higiene oral, por uma restrição motora, falta de motivação e até mesmo falta de orientação profissional, ocorre uma alta incidência de cárie e doença periodontal. Realizou-se uma revisão de literatura, com o objetivo de aprofundar o conhecimento dos cirurgiões dentistas (CD) com relação as características sistêmicas, físicas e em particular das alterações bucais destes pacientes, e com isso facilitar o tratamento desses pacientes no consultório odontológico.

Palavras-chave: Síndrome de Down; Saúde bucal; Odontopediatria; Ensino.
\end{abstract}

\begin{abstract}
Down Syndrome is one of the most periodic chromosomal abnormalities. People who have this syndrome possess numerous oral and functional changes that may have consequences on health care and oral hygiene. There are several systemic diseases associated with this syndrome, making it significant to understand their repercussions on the oral cavity and dental treatment. Due to needy oral hygiene, motor restriction, absence of motivation and even lack of professional suggestion, there is a high incidence of dental caries and periodontal disease. A literature review was carried out on the knowledge of dental surgeons regarding the systemic, physical and in particular oral alterations traits of these patients, therefore promoting the treatment of these patients in the dental clinical.

Keywords: Down Syndrome; Oral health; Pediatric dentistry. Teaching.
\end{abstract}

\section{Introdução}

De acordo com a Organização Mundial de Saúde (OMS) $10 \%$ da população mundial é portadora de algum tipo de deficiência e apenas $3 \%$ recebem atendimento adequado. ${ }^{1}$ Atualmente a uma grande dificuldade dos profissionais na área da odontologia em lidar com pacientes especiais, entre eles, pacientes com Síndrome de Down (SD), que precisam de uma maior orientação e ajuda para uma boa higienização. ${ }^{2}$

Estima-se que a cada 600 nascimentos vivos, um nasce com Síndrome de Down, que é uma alteração genética em que o indivíduo é portador do cromossomo 21 extra, conhecida também como trissomia do 21. Foi descrita por John Langdon Down em 1866, sendo uma das anomalias cromossômicas mais frequentes. ${ }^{3}$

Devido as alterações mentais, motoras, e até mesmo por falta de orientação profissional, muitos desses pacientes possuem uma precária higiene oral, aumentando a incidência de cárie dentária e gengivite. Portanto é de extrema importância um profissional de saúde bucal na reabilitação destes pacientes no meio social. ${ }^{4}$

Este trabalho tem como principal objetivo, revisar e descrever as características bucais presentes em paciente com Síndrome de Down. Deste modo, contribuir para melhor fundamentar os profissionais da área da saúde, com a finalidade de facilitar o tratamento destes pacientes no consultório odontológico. 


\section{Metodologia}

O presente trabalho trata-se de uma revisão da literatura, para o levantamento empregou-se, preferencialmente, a busca de artigos nacionais e internacionais publicados dentre 2001 e 2018, disponíveis na base Google Acadêmico, Scientific Electronic Library Online (SciELO) e PubMed. Foram analisados 17 artigos considerando os aspectos gerais da Síndrome de Down e aspectos específicos como: características bucais, alterações dentais e o papel do cirurgião dentista na manutenção da saúde bucal dos pacientes com essa síndrome.

\section{Revisão de Literatura}

\subsubsection{Síndrome de Down}

A Síndrome de Down (SD) ou também conhecida como trissomia do 21, é uma alteração genética decorrente do cromossomo 21, que ocorre com a alteração dos gametas ou logo após a fecundação inadequada, onde os indivíduos carregam 47 cromossomos. ${ }^{5,6}$

Essa alteração foi descoberta por John Langdon Down em 1866, sendo uma das anomalias cromossômicas mais frequentes. Em 1959 os cientistas Jerome LeJeune e Patricia Jacobs descobriram a causa dessa anomalia, sendo a trissomia do cromossomo $21 .^{6}$

Clinicamente, os pacientes com SD tem défist mental, que varia de criança para criança, e comprometimento no desenvolvimento da linguagem, por terem, na maioria dos casos, alteração bucal. ${ }^{6}$ Além dessas alterações, esses pacientes podem possuir hipotonia muscular, baixa estatura, pescoço curto e largo, orelhas baixas e com aparência dobrada, olhos afastados, entre outras. ${ }^{7,8}$

\subsubsection{Incidência}

A incidência de SD é aproximadamente 3\% da população mundial. ${ }^{3}$ Mulheres que engravidam a partir de 30 anos de idade, o risco de ter um filho com essa alteração genética é de 1:1000 e aumenta para 9:1000 quando a mulher engravida a partir dos 40 anos de idade. ${ }^{9}$

A expectativa de vida desses pacientes pode variar de 35 a 40 anos, mas devido a melhoria no atendimento á saúde, a expectativa de vida aumentou em $80 \%$, podendo esses pacientes viver 55 anos ou mais. ${ }^{7}$

\subsubsection{Características sistêmicas}

Pacientes com SD podem ter algumas alterações sistêmicas presentes como, cardiopatia congênita, problemas de visão e audição, alteração na glândula tireoidea, obesidade, envelhecimento precoce e fator imunológico baixo. ${ }^{5}$ Pode-se ter também o risco elevado de leucemia, diabetes, epilepsia e desenvolvimento de Alzheimer. ${ }^{6}$

\subsubsection{Características Bucais}

São diversas as alterações bucais presentes em paciente com Síndrome de Down, tais como: mandíbula e cavidade bucal pequenas, palato estreito devido a respiração pela boca, o que leva ao palato ogival e profundo, lábios hipotônicos, língua fissurada e grande, podendo apresentar também queilite angular, devido a dificuldade no fechamento da boca. ${ }^{9}$

Quanto as características dentais, podem ser encontrados macroglossia, hipotonia muscular, macrodontia, microdontia, oligodontia, hipodontia, fusão, taurodontia, supranumerários, retenção prolongada dos dentes decíduos, agenesia dental, defeito em esmalte, dentes conóides, anatomia irregular nos dentes, com maior prevalência em dentes permanentes. ${ }^{5,8,10}$

Em pacientes com macroglossia se tem a incomodidade da língua na cavidade bucal, o que causa o deslocamento dos dentes, levando a respiração bucal e uma má oclusão. Como consequência da macroglossia os lábios ficam com muita saliva, levando a irritação, queilite angular, fissuras, facilitando alguns processos infecciosos. ${ }^{6,11}$

$\mathrm{O}$ aumento da língua nesses pacientes pode estar presente devido à hipotonia muscular, pela musculatura mais flácida, levando a uma dificuldade na fala, mastigação e deglutição. Pode haver também a presença de língua fissurada proporcionando a retenção de bactérias e restos alimentares causando a halitose. ${ }^{6,11,12}$

Esses pacientes possuem hipodesenvolvimento da maxila e do palato em relação à mandíbula, causando o encurtamento da arcada superior, desenvolvendo o apinhamento dental e mordida cruzada posterior. A língua dos pacientes com SD protrai os dentes anteriores, provocando assim, a mordida aberta anterior. ${ }^{6,8,10,11}$

Devido à má relação entre maxila e mandíbula eles podem desenvolver uma oclusão tipo II e III de Angle e bruxismo, que é uma das manifestações mais comuns nesses pacientes, pela alta prevalência de anomalias oclusais, o que leva a uma força excessiva, causando o desgaste no dente, deixando-o com uma aparência achatada e de encurtamento, problemas periodontais e sintomatologia dolorosa. $6,8,10,11$

Devido a uma má higienização, a presença de cálculo, macroglossia, e o hábito do paciente em manter a boca aberta, pode favorecer a progressão da doença periodontal. ${ }^{8}$

A doença periodontal é a mais comum em pacientes com SD e tende aumentar com a idade. Devido ao fator imunológico muito baixo, o organismo exibe certa dificuldade em combater as bactérias que estão no biofilme, fazendo com que essa doença seja ainda mais agressiva. Tem o seu diagnóstico precoce, tendo-se a progressão rápida e severa, atinge o tecido de sustentação e de proteção causando a mobilidade e uma reabsorção óssea, sendo o quadrante inferior mais acometido que quadrante superior. ${ }^{6,7,8,10,11,14}$ 
Há uma baixa incidência de cárie nesse pacientes, o que pode estar relacionado a erupção tardia, tendo menos tempo de exposição a fatores de risco, diastemas e bruxismo, que causa um desgaste na superfície oclusal, porém o principal fator é o aumento da capacidade tampão da saliva e saliva alcalina. ${ }^{9,10,11,12,13}$

\subsubsection{Manejo Comportamental}

Pacientes que possuem SD necessitam de uma atenção odontológica com cuidados específicos. Por terem limitações motoras e mentais, apresentam uma dificuldade maior em fazer uma adequada higiene oral. 1,2,15

Atualmente a demanda de pacientes com necessidades especiais que procuram um atendimento adequado é grande, porém, os profissionais que tem dificuldade no manejo desse paciente ainda é enorme. Isso pode se explicar pelo fato do despreparo, e até mesmo o desconhecimento em lidar corretamente com esses pacientes. 1, 4, 7,16

Em alguns casos, os cirurgiões dentistas optam pela anestesia geral, por terem uma grande dificuldade em realizar tratamentos mais invasivos como a cirurgia, ficando mais fácil a reabilitação oral em uma única sessão. Porém esse método só é indicado quando os outros forem ineficientes para o tratamento. ${ }^{16}$

Uma vez o cirurgião dentista tendo o conhecimento sobre as características bucais e comportamentais, é capaz de atuar com mais segurança e presteza, conhecendo as suas limitações e o diferencial que esses pacientes apresentam. ${ }^{1,8,11,17,18,19}$

\section{Resultados e Discussão}

Pacientes com síndrome de Down tem diversas alterações dentais, entre elas encontram-se macroglossia, hipotonia muscular, macrodontia, microdontia, oligodontia, hipodontia, fusão, taurodontia, supranúmerários, retenção prolongada dos dentes decíduos, agenesia dental, defeito em esmalte, dentes conóides, anatomia irregular nos dentes, com maior prevalência em dentes permanentes. ${ }^{5,8,10,14}$ Dentre essas alterações as mais comuns nesses pacientes estão giroversão, com o percentual mais alto $(34 \%)$ agenesia e dente conóide. ${ }^{5}$

Porém Nacamura et al. (2015) afirmam que as alterações mais encontradas foram hipotonia muscular da língua, língua fissurada, dentes conóides, agenesia, entre outros, não sendo mencionado a giroversão. ${ }^{11}$

Alguns autores defendem que o baixo índice de cárie é devido as agenesias que esses pacientes possuem, aumentando os espaços interproximais entre os dentes, dificultando assim o acúmulo de alimentos, porém, há uma outra literatura que explica que, esse índice se da devido a erupção tardia dos dentes, ficando menos expostos as bactérias. ${ }^{11}$ Segundo Vilela et al. (2018), a incidência de cárie é menor devido a capacidade tampão da saliva e a maior tendência a ter bruxismo, desgastando assim a oclusal dos dentes e dificultando a retenção de alimentos. ${ }^{7,13}$

Em relação à língua desses pacientes há controversas na literatura, uma vez que pesquisadores acreditam que pacientes com SD apresentam macroglossia ${ }^{6}$, e outros relatam que a língua possui o tamanho normal, mas como a cavidade bucal que tem o tamanho reduzido devido ao hipodesenvolvimento do terço médio, a língua é posicionada entre os lábios, dando um aspecto de uma língua maior. ${ }^{11}$

As maloclusões mais frequentes observadas foram a mordida aberta e cruzadas posterior, isoladas ou associadas, segundo Manzano et al., porém, difere dos dados obtidos por Bruffaldi et al. que afirma uma maior prevalência de mordida cruzada anterior e maloclusão classe II. ${ }^{12,15}$ Para Santos et al. a maxila apresenta hipodeselvolvimento com retrusão do terço médio, assim explicando a predominância da classe III. ${ }^{4}$

As doenças periodontais são frequentes nesses pacientes, e podem estar associadas a uma pobre higiene oral, anomalia bucofaciais, morfologias dentais, defeito de oclusão e potencial de cicatrização, que é muito baixo para esses pacientes. ${ }^{3,15,17,14}$ Mas para Vilela et al. (2018) a causa dessa alta frequência é um erro no sistema imunológico gerando a diminuição da quimioterapia e fagocitose realizada pelos neutrófilos e monócitos, mudança histológica dos tecidos e efeitos medicamentosos. . $^{8}$

Quando os pacientes com SD são submetidos à anestesia geral podem apresentar algumas complicações por possuírem o pescoço curto e macroglossia, dificultando a incubação traquial. Por esse motivo, também é usado em consultórios odontológicos sedação inalatória consciente (gás de oxido nitroso e oxigênio), no qual o paciente fica consciente, responde a estímulos físico, diminuindo a dor, ansiedade e tem término de sedação rápida, não prejudicando a repercussão do paciente e o profissional após o atendimento. ${ }^{16,20}$

Atualmente no Brasil ainda são poucas as faculdades de odontologia que tem o preparo adequado para o atendimento de pacientes especiais, que requerem cuidados específicos á sua condição. ${ }^{1,2,15}$ Esses pacientes tem uma certa dificuldade em encontrar atendimento odontológico específico, pelo grande número de profissionais despreparados. ${ }^{16}$ Há uma necessidade de proporcionar um maior contato desses pacientes, com os profissionais, pois os mesmos precisam estar atentos e conhecer as características que eles apresentam, criando uma melhor relação e transmitindo segurança ao paciente no atendimento.

\section{Conclusões}

A síndrome de Down é uma alteração cromossômica, no qual os portadores apresentam um elevado índice de alterações sistêmicas, físicas e mentais, sendo mais frequentes problemas periodontais, e um alto índice de alterações bucais como macroglossia, hipotonia muscular, macrodontia, microdontia, oligodontia, hipodontia, fusão, taurodontia, supranumerários, retenção prolongada dos dentes decíduos, agenesia dental, defeito em esmalte, dentes conóides, entre outros, dificultando o tratamento e sendo um desafio para o profissional.

Os profissionais precisam se aprofundar mais nas características bucais que esses pacientes apresentam, que visa, prevenir e controlar não só a doença periodontal, mas também as anomalias que eles possuem ou podem adquirir. 


\section{Referências}

1. Santos MFS, Hora IAA. Atenção odontológica a pacientes especiais: atitudes e percepções de acadêmicos de odontologia. Revista da ABENO 2012; 12(2): 207-12.

2. Oliveira ALBM, Giro EMA. Importância da abordagem precoce no tratamento odontológico de pacientes com necessidades especiais. Odonto 2011; 19(38): 45-51.

3. Ferreira R, Bunduki BO, Teodovich VNJ, Ferreira EAC, Michel RC, Zangrano MSR, et.al. Promoção de saúde bucal e síndrome de Down: inclusão de qualidade de vida por meio de extensão universitária. Odonto 2016; 24(48): 45-53.

4. Pini DM, Frohlich PCGR, Rigo L. Avaliação da saúde bucal em pessoas com necessidades especiais. Einstein 2016; 14(4): 501-7.

5. Santos MR, Oliveira KL, Fonte JBM, Hora IAA, Takeshita WM, Melo MFB. Prevalência de alterações dentárias em pacientes com síndrome de Down avaliados por meio de radiografia panorâmica. Rev. Odontol. Univ. Cid. São Paulo 2014 maio/ago; 26(2): 112-8.

6. Melo CLJA, Dias VM, Almeida NB, Filho PMCC. Síndrome de Down: abordando as alterações odontológicas em pacientes com esta síndrome. Temas em saúde 2017; 17(1): 18-28.

7. Vilela JMV, Nascimento MG, Nunes J, Ribeiro EL. Características bucais e atuação do cirurgião-dentista no atendimento de pacientes portadores de síndrome de Down. Ciências biológicas e de saúde unit. 2018 nov; 4(1): 89-101.

8. Silva FB, Sousa SMG. Síndrome de Down - Aspectos de interesse para o cirurgião-dentista. Saluvista 2001; 20(2): 89-100.

9. Mathias MF, Simionato MRL, Guaré RO. Some factores associated with dental caries in the primary dentition of children with Down syndrome. European journal of paediatric dentistry 2011; 12(1): 37-42.

10. Areias C, Pereira ML, Pérez MD, Macho V, Coelho A, Andrade D, et al. Enfoque clínico de niños con síndrome de Down en el consultorio dental. Avances em odontoestomatol 2014; 30(6): 307-13.

11. Nacamura CA, Yamashita JC, Busch RMC, Marta SN. Síndrome de Down: inclusão no atendimento odontológico municipal. FOL 2015 jan/jun; 25(1): 27-35.

12. Santagelo CN, Gomes DP, Vilela LO, Deus TS, Vilela VO, Santos EM. Avaliação das características bucais de pacientes portadores de síndrome de Down da APAE de Mogi das Cruzes - SP. ConScientiae saúde 2008; 7(1): 29 -34.

13. Scalioni F, Carrada C, Machado F, Devito K, Ribeiro LC, Cesar D, et al. Salivary density of Streptococcus mutans and Streptococcus sobrinus and dental caries in children and adolescents with Down syndrome. J appl oral sci 2017; 25(3): 2507

14. Vieira TR, Péret ACA, Filho LAP. Alterações periodontais associadas às doenças sistêmicas em crianças e adolescentes. Rev. Paul. Pediatr 2010;28(2): 237-4

15. Camara GT, Mascarello AP, Bardini DR, Fracaro GB, Ceranto DCFB. O papel do cirurgião-dentista na manutenção da saúde bucal de portadores de síndrome de Down. Odontol. Clin.-cient. 2011 jul/set; 10(3): 247-250.

16. Andrade APP, Eleutéio ASL. Pacientes portadores de necessidades especiais: abordagem odontológica e anestesia geral. Rev. Bras. Odontol. 2015 jan/jun; 72(1/2): 66-9. 
17. Berthold TB, Araujo VP, Robinson WM, Hellwig I. Síndrome de Down: aspectos gerais e odontológicos. R. Ci. Méd. Biol. $2004 \mathrm{jul} / \mathrm{dez} ; 3(2): 252-60$.

18. Santos, LGP, et al., Manejo do comportamento infantil e saúde bucal na sala de espera: um relato de experiência Research, Society and Development. 2020, 9(7):1-12. e888975069 DOI: http://dx.doi.org/10.33448/rsd-v9i7.5069

19. Shitsuka C, Friggi, MNP, Volpini RMC, Influência dos pais sobre o comportamento infantil no atendimento odontológico. Research, Society and Development. 2020,8(7):e43871154 DOI: http://dx.doi.org/10.33448/rsd-v8i7.1154

20. Silva ZCM, Pagnoncelli SD, Weber JBB, Fritscer AMG. Avaliação do perfil dos pacientes com necessidades especiais da clínica de odontopediatria da faculdade de odontologia da pucrs. Fac. Odonto/PUCRS 2005 out/dez; 20(50): 313-18. 